\section{SCREENING \\ Get it off your chest}

ung cancer is the leading cause of cancer-related death in the world. Many of the symptoms of lung cancer are non-specific and, by the time the patient seeks medical attention, the disease has frequently already progressed to advanced stages. It is, therefore, of critical importance to detect lung cancer at an early stage and, for the past four decades, great effort has been invested in studies that assess the effectiveness of screening and prevention.

Early studies had compared the effect that different modalities of screening (for example, sputum cytology or chest radiographs) had in reducing lung cancer mortality but had failed to show any significant reduction. "These studies were inconclusive because of small sample size, follow-up being too short or too much screening in the control arm (contamination)," explains Philip Prorok- senior investigator of The Prostate, Lung, Colorectal, and Ovarian (PLCO) trial. The lung component of the PLCO trial was designed to compare annual radiographic screening with usual care and to perhaps confirm earlier observations, because as Prorok notes: "there was also the possibility that chest $\mathrm{x}$-ray equipment and/or techniques and lung cancer treatment had improved since the time of the earlier studies". Recently, The National Lung Screening Trial (NLST) showed that screening with low-dose CT resulted in at least $20 \%$ fewer lung cancerrelated deaths compared with screening through chest radiograph. Nevertheless, as this trial chose chest $\mathrm{x}$-ray as a comparator, the results of the PLCO trial were critical to assess the real benefit or harm of CT screening compared with usual care.

The study included 154,901 participants, 77,445 of whom were randomly assigned to receive a chest radiographs at baseline and annually for 3 years (intervention group) and 77,456 participants to receive usual care (usual care group). All participants were 55-74 years old and, as the PLCO trial was designed to assess screening techniques in more than one cancer, there was no selection between smokers or non-smokers. The primary end point was mortality rate and the secondary end points were incidence of lung cancer, cancer stage, survival and potential harms of screening. In the intervention group, 41,403 of the participants followed the regimen up to round three of the screening. Overall adherence was $83.5 \%$ and $91 \%$ of the participants received at least one round of chest radiograph. There was a low contamination rate in the study as only $11 \%$ of the participants in the usual care group underwent a chest radiograph screening. Both groups of participants were followed up for 13 years from randomization.

In the 13 years that the study lasted, there were 1,696 lung cancers detected in the intervention group, although only 505 (30\%) were detected during the 4 years of screening (18\% cancers detected by screening and $12 \%$ during intervals between screening). It is, therefore, difficult to evaluate the overdiagnosis in this group as most of the cancers were not detected through screening; however, as Prorok explains: "the lung cancer incidence rate ratio was 1.05 suggesting a $5 \%$ excess of cases in the screened arm that persisted out to 13 years and showed no indication of diminishing." When the excess cases were compared with the cases detected at screening, the estimated rate of overdiagnosis was $19 \%$. Similar to the intervention group, there were 1,620 cases diagnosed in the usual care group and the stage and histology of the detected tumors was very similar across the two groups.

Regarding whether the study met the primary end point, there were 1,213 lung cancer-related deaths in the intervention group and 1,230 in the usual care group, showing that annual chest radiographic screening for up to 4 years did not significantly reduce lung cancer mortality compared with usual care. "This finding confirms the lack of benefit and overdiagnosis seen in earlier studies," comments Prorok.

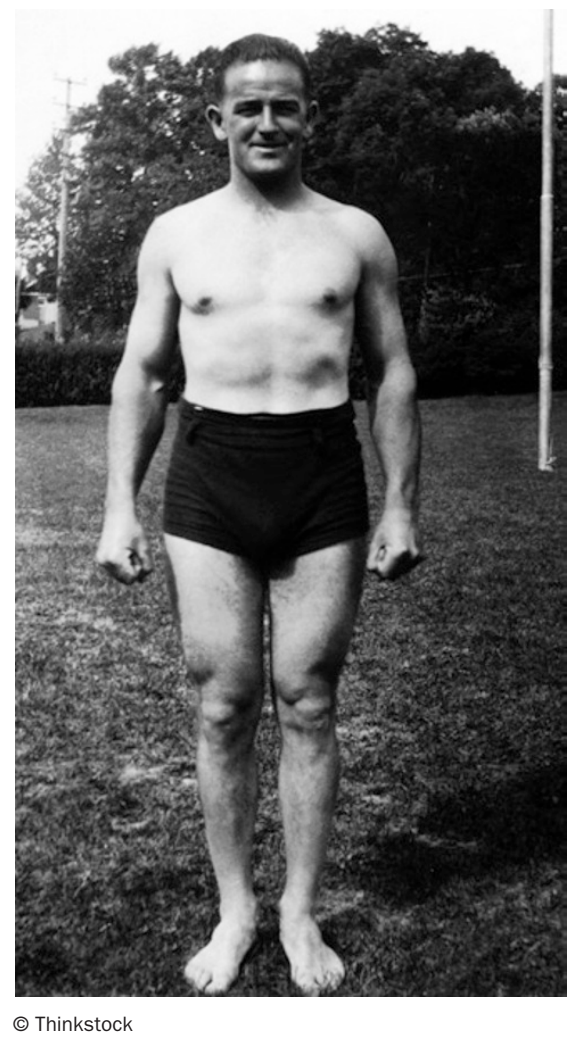

Does this mean that screening should not be recommended as it could do more harm than good? "I personally believe that chest $\mathrm{x}$-ray screening for lung cancer should not be recommended since the evidence shows that there is no benefit but there is harm in terms of overdiagnosis, as well as the false positives that accompany any screening test," explains Prorok. "One could consider spiral CT screening as evaluated in the NLST trial as a screening intervention, although it must now undergo cost-effectiveness evaluation. In addition, the search for better screening tests should be pursued, such as the use of lung cancer biomarkers." For now, it seems that the cheapest and easiest method to prevent lung cancer is to quit smoking!

\section{Teresa Villanueva}

Original article Oken, M. et al. Screening by chest radiograph and lung cancer mortality. JAMA 306, 1916-1918 (2011)

Further reading The National Lung Screening Trial Research Team. Reduced lung-cancer mortality with low-dose computer-tomographic screening. N. Engl. J. Med. 365, 395-409 (2011) 